
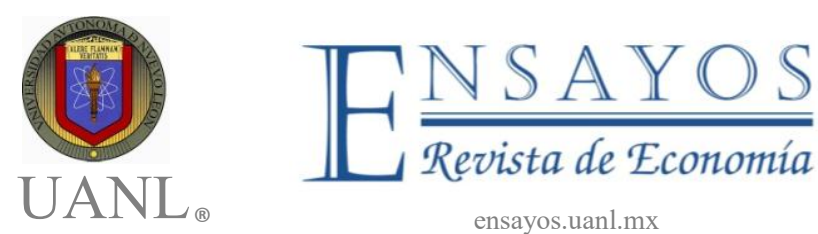

ensayos.uanl.mx

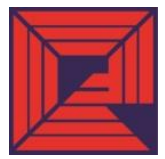

Facultad de Economía

\title{
Opción climática para la producción de café en México
}

\author{
Climate Option of Coffee Production in Mexico
}

\author{
Allou Allou Alphonse* \\ José Carlos Trejo García \\ Miguel Ángel Martínez García
}

\begin{tabular}{l}
\hline Article information \\
\hline Received: \\
1 August 2017 \\
Accepted: \\
27 July 2018 \\
\hline JEL Classification: \\
C52 ; D83 ; G12 ; G24 ; \\
Q14
\end{tabular}

Keywords: Climatic Option; Weather Derivatives; Coffee Production

\begin{abstract}
This paper presents weather derivatives as a tool for the agricultural sector in Mexico to protect itself from climatic risks. An analysis of the need for a climate derivative for the cultivation of coffee in the states of Veracruz, Chiapas and Oaxaca in order to propose a derivative for transferring risk associated with climate. Therefore, it was determined using the data panel method that precipitation has a 1.99 elastic relationship with the annual production of coffee. On the basis of this argument, a valuation is developed in this study of European options related to precipitation and coffee using the Black-Scholes method (1973) for the states mentioned.
\end{abstract}

\footnotetext{
*Escuela Superior de Economía, SEPI, Instituto Politécnico Nacional. allou_alfonse@yahoo.fr

${ }^{* *}$ Escuela Superior de Economía, SEPI, Instituto Politécnico Nacional. jtrejog@ipn.mx

${ }^{* * * *}$ Escuela Superior de Economía, SEPI, Instituto Politécnico Nacional. mmartinezga@ipn.mx
} 


\section{Información del} artículo

Recibido:

1 agosto 2017

Aceptado:

27 julio 2017

Clasificación JEL:

C52 ; D83 ; G12 ; G24 ; Q14

Palabras Clave:

Opción climática;

Derivados climáticos;

Producción de Café

\section{Resumen}

En este trabajo, se presentan los derivados climáticos como un instrumento que permite al sector agrícola en México cubrirse ante los riesgos relacionados con el clima. Se realiza un análisis de la factibilidad de un derivado climático para el cultivo del café en los estados de Veracruz, Chiapas y Oaxaca con el fin de transferir los riesgos asociados con el clima. Así se determina, mediante el método de panel de datos, que la precipitación tiene una relación elástica de 1.99 con la producción anual del café. Partiendo de este argumento, se desarrolla en el presente trabajo una valuación de opciones europeas sobre precipitación para el café con el método de Black-Scholes (1973), para los estados estudiados.

\section{Introducción}

El calentamiento global y los fenómenos derivados juegan un papel importante en la actividad humana. En diversos sectores de la economía, el fenómeno mundial del cambio climático y los riesgos asociados están omnipresentes. En efecto, es importante saber qué se entiende con el concepto de cambio climático. Se deben diferenciar el cambio climático y el concepto de variabilidad del clima.

Parry M.L., O.F. Canziani, J.P. Palutikof, P.J. Van der Linden y C.E. Hanson (2007), definen el clima como las condiciones promedio del tiempo atmosférico en un periodo que es generalmente de 30 años y, a su vez, presentan el cambio climático como la modificación en el comportamiento promedio del clima durante un periodo largo. De acuerdo con Agrawala (2005), hablar de la variabilidad climática es hacer referencia a las variaciones relacionadas con el clima en las escalas temporal y espacial como las sequías e inundaciones, el fenómeno El Niño (ENSO) ${ }^{1}$ y la incidencia de frentes fríos, entre otros.

Dentro de los sectores económicos afectados por el cambio climático y sus variaciones, se encuentra el agrícola. En México, como en la mayoría de los

\footnotetext{
${ }^{1}$ El Niño Southern Oscilaton, por sus siglas en inglés (ENSO), es un fenómeno climático cíclico que provoca estragos en el nivel mundial. Pueden observarse cambios en la dirección y en la velocidad de los vientos, así como en el desplazamiento de las zonas de lluvia a la región tropical.
} 
países en desarrollo, la población de escasos recursos que vive directamente de la agricultura, es la más afectada por este fenómeno natural, ya que los cambios que se generan en la temperatura y en las precipitaciones tienden a afectar las condiciones agroclimáticas, las épocas de siembra, etc. (GTZ., 2010).

Rosenzweig, C., Iglesias, A., Yang, X.B., Epstein, P.R. y Chivian, E. (2001), estiman que la agricultura es un sector que tiene sus ingresos altamente correlacionados con elementos como la temperatura, la precipitación, la humedad y la luz solar. Especialmente, si el cambio climático aumenta las fluctuaciones meteorológicas, como se espera en el futuro cercano (Easterling, D.R., Evans, J.L., Groisman, P.Y., Karl, T.R., Kunkel, K.E. y Ambenje, P., 2000).

Para el cultivo del café, Houghton et al. (2001), mencionan que existe una mayor variación en las condiciones climáticas cada año, y que la presencia de climas más extremos con un incremento en la frecuencia de aparición o de duración del fenómeno de El Niño, puede provocar mayores fluctuaciones año con año, tanto en la cantidad, como en la calidad de la cosecha. Jaramillo, Muchugu, Vega, Davis, Borgemeister y Chabi-Olaye (2011) consideran que los problemas ecológicos más importantes que enfrenta el mundo, son el cambio climático y las especies invasoras. Por lo tanto, se espera que el cambio climático pueda afectar la productividad de diferentes cultivos agrícolas, incluyendo el café.

Por su parte Gay et al. (2006), exploran la relación entre la producción de café en función de las variables climáticas y económicas del estado de Veracruz, con un modelo económico; ellos encuentran que, en la mitad del siglo XXI, se espera una reducción de la productividad del café entre $22 \%$ y $27 \%$ a causa de un posible incremento de la temperatura y de una posible disminución de la precipitación. De igual manera, Gay y coautores, en su estudio sobre la relación entre la producción de café y las variables climáticas y económicas en Veracruz, México, para 2020, afirman que se espera una reducción de la precipitación de 33,48 milímetros $(\mathrm{mm})$ y un pequeño aumento de la temperatura media $(0,01 \%)$, con una disminución de $24,54 \%$ en la producción.

Villers, L., Arizpe, N., Orellana, R., Conde, C. y Hernández, J. (2009), quienes hicieron una evaluación del impacto del cambio climático sobre el florecimiento y desarrollo del café arábigo en la región de Huatusco, Veracruz, encontraron que el cambio de temperatura del aire puede alterar la floración, y que el cambio de las variaciones de agua, durante el crecimiento del fruto, puede afectar de manera significativa su rendimiento. Por lo anterior, es imperativo administrar de manera eficiente dichos riesgos, 
particularmente en el sector agrícola de México. Frente a dichos riesgos en este sector, se propone la utilización de los derivados climáticos ${ }^{2}$ como instrumentos de cobertura, ante una posible pérdida en la rentabilidad por causa de eventos climáticos.

Los derivados climáticos son herramientas de transferencia de riesgo que combinan de forma apropiada el riesgo del clima y la rentabilidad deseada. Turvey, C.G. (2001), menciona que, con el uso de los derivados climáticos como instrumentos de cobertura, se puede asegurar la rentabilidad de un cultivo agrícola. Jewson, S. y Brix, A. (2005), por su parte, presentan la cobertura con derivados del clima como una buena opción por diversas razones. En efecto, cubrirse con este instrumento permite la disminución de la volatilidad en los beneficios de la empresa, lo que se puede traducir en una reducción de la tasa de interés que enfrenta la empresa; además, se puede observar la disminución de la volatilidad de las acciones de una compañía que cotiza en bolsa, la reducción de la volatilidad de sus ingresos y por tanto, su calidad. Generando así, una revaloración de las acciones de la empresa y la disminución de la volatilidad en los beneficios, con lo cual se reduce el riesgo de quiebra.

En la actualidad, el mercado financiero mexicano es un mercado en desarrollo y todavía restringido en comparación con los mercados financieros de Estados Unidos, Europa y Japón. En México, uno de los mayores problemas que ha enfrentado y sigue enfrentando el sector agropecuario desde hace muchos años, es la incertidumbre sobre el valor de su producción a causa del clima. De esta forma, estos derivados son una alternativa de cobertura financiera frente a eventos meteorológicos altamente probables, conocidos como eventos climáticos no catastróficos (sequías, exceso de lluvias, temperaturas extremadamente altas o bajas, etc.), para empresas o productores agrícolas, cuyas utilidades están correlacionadas con las condiciones meteorológicas.

Por tales razones, este trabajo presenta la valuación de la factibilidad económica -financiera de diseñar, usar y comercializar contratos de opciones sobre el clima para los productores de café, en los estados de Veracruz, Chiapas y Oaxaca. Asimismo, se valuó una opción europea sobre precipitación como instrumento de cobertura de la rentabilidad del café en los estados de Veracruz, Chiapas y Oaxaca, con el objetivo de mitigar los riesgos

\footnotetext{
${ }^{2}$ Los derivados tradicionales radican en un subyacente negociable, como son los precios de activos financieros, bonos y commodities; por otro lado, el subyacente de un derivado climático es un parámetro climático que se apoya en el clima mismo y que puede medirse objetivamente como la temperatura promedio, precipitaciones, velocidad del viento, etc., todos en un período y lugar determinado.
} 
climáticos asociados con la actividad agrícola. Por tanto, este trabajo se divide en seis partes.

Después de la introducción, se hace una revisión de la literatura sobre los derivados climáticos en la agricultura. En tercer lugar, se ofrece una breve presentación del café en México y en cuarto término, se aplica el método de panel de datos con un modelo de producción Cobb-Douglas adaptado a la agricultura, con la metodología de Cruz y Llinás (2010), con el fin de determinar cuál factor climático afecta más la rentabilidad del café para los estados de Chipas, Veracruz y Oaxaca ${ }^{3}$. Posteriormente, se calculó con base en el modelo de valuación propuesta por Cruz y Vargas (2008), el derivado para el factor climático correspondiente con el modelo de Black-Scholes $(1973)^{4}$, que servirá a la valuación del derivado climático; finalmente, se presentan las conclusiones.

Este trabajo contribuirá a mostrar la factibilidad económico-financiera de diseñar, usar y comercializar contratos derivados climáticos para los productores de café, en los estados de Veracruz, Chiapas y Oaxaca. La cobertura por medio de los derivados puede servir a la mitigación de los riesgos originados por el clima en el sector agrícola, en México. Por lo tanto, se puede producir certidumbre para la administración de riesgos, aprovechando sus ventajas y el gran potencial de desarrollo que tienen para la protección contra los riesgos adversos del clima, mediante la transferencia de dichos riesgos al mercado.

\section{Derivados climáticos en la agricultura}

En los años noventa, nace una nueva clase de instrumentos para la transferencia del riesgo financiero relacionado con el clima, que incluye la participación de varios agentes llamados derivados climáticos. Los derivados ${ }^{5}$ climáticos son activos comercializados en los mercados regulados $\mathrm{o}$ estandarizados como en el Chicago Mercantile Exchange (CME) y en los mercados extrabursátiles OTC $^{6}$. Dichos derivados permiten proteger a las empresas de la incertidumbre que genera el clima en sus negocios. La

\footnotetext{
${ }^{3}$ Los estados de Veracruz, Chiapas y Oaxaca son de los más grandes productores de café en México y constituyan cerca del $80 \%$ de la superficie sembrada, según la SIAPSAGARPA (2014).

${ }^{4}$ El Modelo de Black-Scholes-Merton permite calcular el precio de un activo subyacente con la particularidad de reflejar un apego a situaciones reales; sin embargo, como todo modelo matemático, este es simplificado por algunos supuestos.

${ }^{5}$ Un derivado financiero, es un instrumento cuyo valor deriva de un activo subyacente (acciones, índices bursátiles, valores de renta fija, tipos de interés o también materias primas).

${ }^{6}$ Over-the-counter (OTC) es un mercado extrabursátil o mercado de contratos hechos a la medida, donde se negocian instrumentos financieros directamente entre dos partes.
} 
principal diferencia entre los derivados climáticos y los tradicionales, radica en el subyacente, que es el clima, el cual no es un activo negociable, como sí lo son los precios de activos financieros, bonos y commodities ${ }^{7}$.

Además, el subyacente es un parámetro climático que se apoya en el clima mismo y que puede medirse objetivamente, como: la temperatura promedio, precipitaciones, velocidad del viento, etc., todos en un periodo y lugar determinado.

En efecto, los derivados climáticos se presentan como una forma de cubrirse de los efectos del clima. Por lo tanto, los derivados climáticos se han definido sobre las variables climáticas, entre ellas, la temperatura, niveles de lluvia, velocidad y dirección del viento, índices de humedad ambiental, cantidad de horas de sol, etc. Por ejemplo, para comprar una opción, los compradores tienen que pagar una prima, que es el precio de la opción. A largo plazo, la opción de compra tiene un valor positivo si el subyacente tiene un precio superior a lo acordado. Respectivamente, las opciones de venta, tienen un valor positivo si el subyacente es inferior al precio del ejercicio pactado (Jewson y Brix, 2005). De acuerdo con González y Nave (2010), la variable climática no tiene un valor monetario en sí mismo; pero, en el momento de definir el monto del contrato, se debe tener en cuenta el valor que tenga la variable elegida y también se debe especificar una relación entre el índice climático y el valor económico.

Los seguros y los derivados climáticos son herramientas que ayudan a mitigar las pérdidas asociadas con el estado del tiempo o del clima. Los derivados sirven como cobertura ante riesgos de alta probabilidad de ocurrencia, pero con una baja prima, contrariamente a los seguros tradicionales (De Paz Cobo, S., 2004). En primer lugar, los derivados climáticos compensan la incertidumbre de las condiciones meteorológicas, independientemente del daño real, mientras que el seguro tradicional, paga después de ocurrido el evento (Barnett y Mahul, 2007; Fuchs y Wolff, 2011). Por lo general, los seguros tradicionales ofrecen protección contra eventos climáticos extremos que tienen una pequeña probabilidad de ocurrir, contrariamente a los derivados climáticos. En segundo lugar, la rentabilidad de los contratos se mide mediante un índice estandarizado. Stoppa y Hess (2003) estiman que esto reduce los costos de transacción y administración de los participantes. En tercer lugar, Gronberg y Neilson (2007), mencionan el hecho de que el índice climático sólo depende de los factores meteorológicos, los cuales pueden eliminar problemas de riesgo moral, independientemente del comportamiento individual de cada participante.

7 Los commodities, son productos uniformes y estandarizados con muy poca diferenciación, los cuales típicamente son clasificados como materia prima a la cual el mercado le asigna un valor. 
Además, los contratos de derivados climáticos ofrecidos por la bolsa de valores están estandarizados, lo que disminuye aún más los costos de transacción. En la última década, se desarrollaron varias formas para modelar el índice de temperatura y se estudió principalmente el derivado climático basado en la temperatura (Alaton, P., Djehiche, B., Stillberger, D., 2002; Benth y Saltyte, 2007; Schiller, F., Seidler, G. y Wimmer, M., 2012). Vedenov y Barnett (2004) investigaron la cobertura de la temperatura y de la lluvia con los derivados climáticos para el maíz, el algodón y la soya en los Estados Unidos de América, e indican que los derivados climáticos, generalmente pueden reducir el riesgo climático para los agricultores.

Sin embargo, la eficiencia difiere mucho entre cultivos y zonas de cultivos. En los países en desarrollo, una proporción mayor de la población trabaja como agricultores y los ingresos son generalmente más bajos (Dang, 2006). Estas razones aumentan la demanda de los agricultores por mecanismos eficientes de transferencia de riesgo (Wang, Karuaihe, Young, y Zhang, 2013). Si los ingresos son bajos en un año, no son suficientes debido a los bajos rendimientos. Los instrumentos de mitigación del riesgo que reducen las fluctuaciones de los ingresos permiten a los agricultores escapar de la pobreza crónica (Wang et al., 2013).

La introducción de derivados financieros del clima en los países en desarrollo es un proceso difícil, pero su uso puede apoyar al fortalecimiento del sector agrícola. Wang et al. (2013), en su investigación sobre la demanda de seguro climático para la producción de maíz en Sudáfrica, informan que estos productos financieros pueden servir como herramientas eficientes de gestión de riesgos cuando los factores meteorológicos pueden explicar satisfactoriamente el rendimiento.

En México, son comercializados contratos futuros y opciones en la Bolsa de Derivados de México (MexDer), pero todavía no existen derivados del clima para el mercado mexicano. Sería una buena opción comercializar este tipo de productos, ya que existen las estadísticas del clima y una cámara de compensación ASIGNA ${ }^{8}$. Para el café, es posible comercializar este tipo de productos para los estados de Veracruz, Chiapas y Oaxaca, y eso se puede observar por la importancia que tiene el café, tanto para los agricultores como para la economía.

${ }^{8}$ ASIGNA, es la Cámara de Compensación y Liquidación del Mercado Mexicano de Derivados (MexDer). Su principal función es ser la contraparte y, por tanto, garante de todas las obligaciones financieras que se derivan de la operación de los contratos negociados. 


\section{La cultura del café en México}

En México, los huracanes tienden a afectar de manera desastrosa diversos aspectos de la vida. Se han observado muchos desastres y devastaciones a causa de dicho fenómeno, el cual ocasionó severas pérdidas económicas. En 2014, el huracán Odile de categoría 3, fue el huracán más devastador de la temporada de lluvias de dicho año en México. Por lo tanto, las aseguradoras pagaron alrededor de 7 mil millones de pesos para cubrir los daños de acuerdo con la estimación de la Comisión Nacional de Seguros y Fianzas (CNSF). Además de los huracanes, existen otros fenómenos climáticos que causan tales niveles de desastres, como el frente frío número 12 del mes de noviembre de 2014, que provocó intensas lluvias y dejó como damnificados a más de 85,000 personas en los estados de Veracruz y Tabasco. La ocurrencia de dichos eventos no exenta el futuro de los mismos, ya que el estado de Veracruz, una vez más, fue víctima de las fuertes lluvias, inundaciones y deslizamiento del 9 al 13 de julio de 2015.

Para hacer frente a estos eventos catastróficos, se creó en 1996, el Fondo de Desastres Naturales (FONDEN), como un instrumento financiero del Sistema Nacional de Protección Civil (SINAPROC), con el fin de apoyar a las entidades federativas, las dependencias y entidades de la Administración Pública Federal (APF), ante daños causados por fenómenos naturales. Así, el FONDEN hizo frente a diversos desastres de manera histórica. Del mes de enero al mes de septiembre de 2014, fue autorizado un presupuesto de 22 mil 308 millones de pesos para hacer frente a eventuales desastres.

En efecto, en el año 2005, se emitieron, por medio de la Secretaría de Hacienda y Crédito Público (SHCP), las Reglas de Operación de los Programas del Subsidio a la Prima del Seguro Agropecuario y de Apoyo a los Fondos de Aseguramiento Agropecuario, cuyo objetivo es apoyar a los Productores Agropecuarios para reducir el costo de las primas pagadas a los Seguros Agropecuarios contratados. En 2013, se emitió una nueva versión de dichas reglas, donde se puede otorgar el beneficio a Productores Agropecuarios que aseguren cultivos, cuando contraten y paguen la Prima o Cuota de una o varias de las coberturas de riesgo, entre los que se encuentra el riesgo climatológico.

Dentro de los cultivos afectados por la variación de clima, se tiene el café, que es uno de los más importantes cultivos tropicales para los países en desarrollo y los países desarrollados, como Estados Unidos, Europa y Japón, que consumen el $80 \%$ del café producido (Moguel y Toledo, 1999). 
El café se cultiva principalmente en 16 estados de México. De acuerdo con la SAGARPA $^{9}$ (2015), el café representa una actividad estratégica para México, ya que emplea a más de 500 mil productores en alrededor de $690 \mathrm{mil}$ hectáreas sembradas en las 16 entidades federativas donde se produce. Además, vincula directa e indirectamente a cerca de 3 millones de personas, y genera un valor en el mercado de alrededor de 20 mil millones de pesos por año, con dos tipos de café cultivados : el arábica (con aproximadamente 98\%) y el robusta.

En efecto, los estados de Veracruz, Chiapas y Oaxaca son los estados con mayor producción de café, cerca del $80 \%$ de la producción en México. Según la $\operatorname{SIAP}^{10}$ (2014), de 2007 a 2012, dichos estados recibieron cerca del $80 \%$ del apoyo al fomento productivo del café. Además, estos estados tienen el $78 \%$ de las superficies de café sembradas, equivalentes al $78 \%$ de los beneficiarios del apoyo. La producción de café se agrupa en cuatro regiones, que son:

La vertiente del Golfo: San Luis Potosí, Hidalgo, Puebla, México y Veracruz; La vertiente del Océano Pacífico: Colima, Guerrero, Jalisco, Nayarit y parte de Oaxaca;

Región Soconusco: Gran proporción del estado de Chiapas y

Región Centro Norte de Chiapas.

En la siguiente gráfica, se puede observar la relación que existe entre la producción de café, la precipitación y la temperatura para los Estados de Veracruz, Chiapas y Oaxaca.

\section{Gráfica 1}

Producción anual de café 2004-2016 (miles de toneladas)

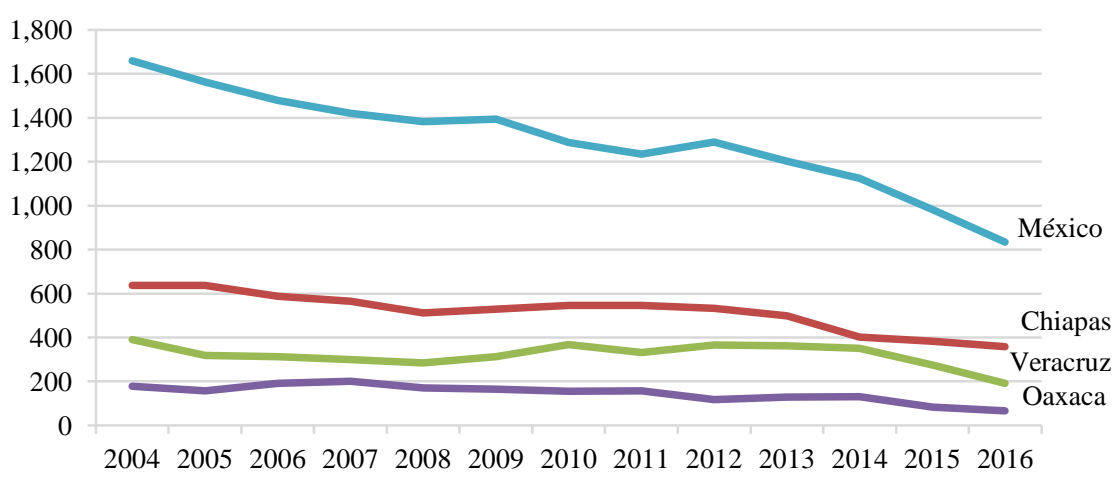

Fuente: Elaboración propia con información de SIAP-SAGARPA 2004-2016.

\footnotetext{
${ }^{9}$ Secretaría de Agricultura, Ganadería, Desarrollo Rural, Pesca y Alimentación de México.

${ }^{10}$ Servicio de Información Agroalimentaria y Pesquera de México
} 
En dicha gráfica, se observa cómo la producción de café ha caído a la mitad en el periodo de 2004-2016. Esto se puede explicar por diversas razones: los hongos, el bajo precio del café y las variaciones del clima, lo que causa que los productores abandonen este tipo de producción por otros cultivos.

Améndola, Castillo y Martínez (2005) afirman que más del $70 \%$ de los cafetales mexicanos se encuentran arriba de los 600 metros de altitud y con una diversidad de microclimas. Se puede observar un clima cálido, donde la temperatura oscila entre $22^{\circ} \mathrm{C}$ y $26^{\circ} \mathrm{C}$ y las precipitaciones oscilan entre $1.000-2.000 \mathrm{~mm}$; mientras que con un clima templado, se presentan temperaturas entre $18^{\circ} \mathrm{C}$ y $22{ }^{\circ} \mathrm{C}$ y precipitaciones que varían de 600 a 1.000 $\mathrm{mm}$, lo que permite obtener un café de calidad. En efecto, el café es una planta que requiere un clima cálido pero con alto nivel de humedad. La planta de café no debe recibir directamente el sol, razón por la cual se siembran distintos árboles junto a los cafetos para generar sombra. La altitud ideal para su buen desarrollo es entre los mil y mil trescientos metros sobre el nivel del mar, pero también se puede producir en mayores alturas e incluso en las costas.

Cabe mencionar que la calidad de la tierra es muy importante, por lo tanto un suelo rico y húmedo permitirá la buena absorción del agua y el rápido drenaje de las lluvias. Vaast, P., Bertrand, B., Perriot, J-J, Guyot, B., Genard, M. (2006) mencionan que un clima más cálido o altitudes más bajas provocan una maduración más rápida de la cereza del café, lo cual afecta la calidad.

Existen diversas razones que posiblemente explican la disminución de la producción de café, la principal radica en las variaciones del clima: tema de interés de este trabajo. Así, mediante un modelo econométrico que utiliza una función de producción de Cobb-Douglas modificada, se busca saber si la temperatura o la precipitación afecta la producción de café en los estados de Veracruz, Chiapas y Oaxaca. Y, posteriormente, con los resultados obtenidos, se pretende saber si los caficultores pueden apoyarse en una oferta de derivado climático específica, que pueda cubrir sus necesidades.

\section{Modelo econométrico}

Se utilizó una función de producción Cobb-Douglas para determinar qué niveles de precipitación o temperatura afectan la producción agrícola de café en los estados de Veracruz, Chiapas y Oaxaca. Se espera con este modelo estimar la sensibilidad de la productividad del café en dichos estados, con respecto a la temperatura y a la precipitación. Cruz y Llinás (2009) modelaron la producción agrícola mediante la función de producción CobbDouglas, con una modificación del modelo original, donde el modelo clásico 
mide la producción con una función que tiene por variables de entrada el Capital $(K)$ y el Trabajo $(L)$.

En este trabajo, las variables exógenas que se asumen son la temperatura y las precipitaciones, y se consideran como constantes los otros factores (ceteris paribus). Por lo tanto, la función de producción modificada permite medir los efectos marginales de las precipitaciones y de la temperatura sobre los niveles de producción.

La productividad marginal del clima es:

$$
Y=A P^{\alpha} T^{\beta}
$$

Donde, $Y$ es la producción de la cosecha de café anual en miles de toneladas, $A$ es el intercepto con el eje de las ordenadas, $P$ representa las precipitaciones acumuladas anuales en milímetros, $T$ es la temperatura promedio anual registrada para el periodo de estudio 2004-2016, la cual se expresa en grados Celsius $\left({ }^{\circ} \mathrm{C}\right), \alpha$ y $\beta=(1-\alpha)$ son los coeficientes de los niveles de precipitación y de temperatura, respectivamente. Se necesita hacer una transformación lineal para estimar el modelo de Mínimos Cuadrados Ordinarios (MCO).

$$
\ln Y=\ln A+\alpha \ln P+\beta \ln T
$$

Donde, $\alpha$ y $\beta$ representan la elasticidad parcial del producto o rendimiento de la cosecha con respecto a los niveles de precipitación y temperatura, respectivamente.

Se calculan las elasticidades a partir de la ecuación (1). Se obtiene lo siguiente:

Derivando parcialmente con respecto a la precipitación:

$$
\frac{\partial Y}{\partial P}=\alpha \frac{Y}{P}
$$

Derivando parcialmente con respecto a la temperatura:

$$
\frac{\partial Y}{\partial T}=\beta \frac{Y}{T}
$$

Por la derivada cruzada con respecto a la precipitación y la temperatura, se tiene:

$$
\frac{\partial^{2} Y}{\partial P \partial T}=\alpha \beta \frac{Y}{P}
$$


Para justificar la efectividad de un derivado climático como instrumento de cobertura del riesgo climático, las ecuaciones (3), (4) y (5) deben ser superiores a cero. Se debe entonces verificar estas condiciones.

$$
\frac{\partial Y}{\partial P}>0 ; \frac{\partial Y}{\partial T}>0 ; \frac{\partial^{2} Y}{\partial P \partial T} \geq 0(6)
$$

$\mathrm{Si} \frac{\partial^{2} Y}{\partial P \partial T}>0$, entonces, tanto la precipitación (lluvia) como la temperatura (el calor o frío) impactan de manera conjunta la producción.

$\mathrm{Si} \frac{\partial^{2} Y}{\partial P \partial T}=0$, entonces, ni la precipitación (lluvia) ni la temperatura (el calor o frío) tienen efecto alguno sobre la producción.

En caso de no cumplimento de dichas condiciones, el clima no tendrá entonces ninguna incidencia sobre la producción agrícola del café; por lo tanto, no hay necesidad de transferir los riesgos asociados por medio de un derivado climático. Así, para comprobar la condición antes mencionada, se hace un análisis econométrico sobre las diferentes variables, con la información de la producción anual del café en los estados de Veracruz, Chiapas y Oaxaca obtenida de la SIAP, de las precipitaciones acumuladas anuales, y de las temperaturas promedio anuales obtenidas de la CONAGUA $^{11}$ para el periodo 2004-2016. Dicho análisis se realiza mediante una estimación de mínimos cuadrados ordinarios, o pooled en datos panel.

Se hacen dos variaciones de panel de datos. Primero, se estima el modelo mediante efectos fijos ${ }^{12}(F E)$, esto para conocer en promedio la producción del café dependiendo de las situaciones climáticas. Después, se aplica una prueba $F$, para ver si las variables sostienen el modelo. Segundo, se hace la estimación del modelo mediante efectos aleatorios ${ }^{13}(R E)$, con la siguiente especificación: las diferencias entre los factores climáticos se capturan a través de un término de perturbación $\omega_{i t}$, que sigue $\omega_{i t}=u_{i}+\varepsilon_{i t}$ donde $u_{i}$ es un término no observable que representa el componente de error específico individual y $\varepsilon_{\text {it } 14}$ que combina la serie de tiempo con el corte transversal.

Dicho análisis se utiliza para buscar los efectos aleatorios mediante la prueba de Breusch-Pagan de Lagrange Multiplicator (BPLM) (1979). Si se rechaza

\footnotetext{
${ }^{11}$ CONAGUA, Comisión Nacional del Agua de México.

${ }^{12}$ Este método considera que existe un término constante diferente para cada individuo y supone que los efectos individuales son independientes entre sí, en los datos.

${ }^{13}$ A diferencia del modelo de efectos fijos, se considera que los efectos individuales no son independientes entre sí, sino que están distribuidos aleatoriamente alrededor de un valor dado.

${ }^{14}$ Corresponden al error asociado con las series de tiempo.
} 
la hipótesis nula ${ }^{15}$ de BPLM, se tiene que H0: $\sigma_{u}^{2}=0$, entonces se tiene efectos aleatorios en el modelo. En la tabla 1, se presentan los resultados obtenidos de las distintas especificaciones.

\section{Tabla 1}

Estimación de la función de producción con Panel de Datos

\begin{tabular}{lccc}
\hline Ly & MCO & FE & RE \\
\hline \multirow{2}{*}{ Lp } & 1.993 & 0.725 & 1.993 \\
& $(0.000)^{* * *}$ & $(0.005)^{* * *}$ & $(0.000) * * *$ \\
Lt & -1.851 & 0.46 & -1.851 \\
& $(0.094)^{*}$ & $(0.59)$ & $(0.085)$ \\
\hline \multirow{2}{*}{ Prueba F } & 42.62 & 24.64 & \\
& $(0.000)^{* * *}$ & $(0.000)^{* * *}$ & \\
\hline
\end{tabular}

Breush-Pagan

Hausman

$\mathbf{R}^{\mathbf{2}} \quad 0.7031$

Fuente: Elaboración propia con datos de la Secretaría de Agricultura, Ganadería, Desarrollo Rural, Pesca y Alimentación de México y del Servicio de Información Agroalimentaria y Pesquera de México. Nota: $(* * *)$ y $(*)$ corresponden a un nivel de confianza de $99 \%$ y $90 \%$, respectivamente.

Para el modelo MCO y bajo la consideración de la ecuación (2), se observa la elasticidad de la precipitación $(P)$ y producción $(Y)$ durante el periodo de 2004-2016. La elasticidad de $P$ tiene un coeficiente de 1.993, significativo al $99 \%$. Hay una relación entre precipitación $(P)$ y producción $(Y)$. Para el caso de la temperatura $(T)$, se observa que el efecto promedio con respecto a la producción $(Y)$, céteris páribus, es de -1.851 , significativa al $90 \%$. La variación de la temperatura tiene una incidencia directa sobre los niveles de producción del café. Se puede interpretar que cuando aumenta la temperatura, entonces, baja la precipitación. El coeficiente de determinación ( $R$-squared) de 0.703 indica en qué proporción la variable dependiente es explicada por las variables independientes, la cual muestra una relación muy estrecha.

Por otro lado, la estimación del modelo econométrico con panel de datos con efectos fijos y efectos aleatorios, permiten observar que la variable precipitación $(P)$ es estadísticamente significativa a diferencia de la variable temperatura $(T)$. La prueba Hausman presenta una probabilidad menor a 0.05 ,

${ }^{15}$ La hipótesis nula: la varianza individual específica es cero. 
entonces hay diferencia sistemática entre los efectos fijos y los efectos aleatorios. Por lo tanto, el modelo de efectos aleatorios no es el adecuado, y se concluye que el mejor ajuste obtenido con el panel de datos, es el que considera efectos fijos. Los resultados anteriores permiten decir que las variaciones en la producción del café son explicadas por las precipitaciones y no por la temperatura. De esta manera, se puede desarrollar un derivado sobre la precipitación, para transferir el riesgo de dicha precipitación, y que esto beneficie a los caficultores de Veracruz, Chiapas y Oaxaca.

\section{Opción climática sobre precipitación para el café en Veracruz, Chiapas y Oaxaca}

En este trabajo, se utiliza el modelo de Black-Scholes (1973) con una simulación Monte Carlo, para el cálculo de la opción climática. La valuación del precio de cada opción se hace con las fórmulas siguientes:

$$
\begin{gathered}
C=\delta\left(S N\left(d_{1}\right)-\delta K e^{-r T} N\left(d_{2}\right)\right)(7) \\
P=\delta\left(K e^{-r T} N\left(d_{2}\right)-\delta S N\left(d_{1}\right)\right)(8)
\end{gathered}
$$

Donde $\mathrm{N}$ representa la distribución normal acumulada, y $\mathrm{d}_{1} \mathrm{y} \mathrm{d}_{2}$ definidas como:

$$
\begin{array}{r}
d_{1}=\frac{\left(\log \left(\frac{S}{E}\right)+\left(r+\frac{1}{2} \sigma^{2}\right)(T-t)\right)}{\sigma \sqrt{T}-t}(9) \\
d_{2}=d_{1}-\sigma \sqrt{T}=\frac{\left(\log \left(\frac{S}{E}\right)+\left(r-\frac{1}{2} \sigma^{2}\right)(T-t)\right)}{\sigma \sqrt{T}-t}(10)
\end{array}
$$

C: Call sobre precipitaciones.

P: Put sobre precipitaciones.

S: Precipitación acumulada anual, en milímetros, a la fecha del 31 de diciembre de 2016.

K: Precipitación acumulada anual promedio, sobre el periodo de estudio (2004-2016).

$\mathrm{r}$ : Tasa libre de riesgo de corto plazo (Cetes $91^{16}$ ), obtenida del Banco de México.

T: Tiempo de maduración del contrato (un año).

$\delta$ : Volatilidad del subyacente.

$\mathrm{t}$ : Momento del tiempo menor a $\mathrm{T}$.

Las ecuaciones (7) y (8) son la solución cerrada para la valoración de opciones climatológicas europeas ${ }^{17}$ con el método de Black-Scholes.

\footnotetext{
${ }^{16}$ El contrato se hace por un corto plazo, por lo que -en este caso- se toma el valor del CETES 91 por un año de contrato.

${ }^{17}$ Con una opción europea, el contrato que se ejerce a vencimiento.
} 
Este cálculo se realiza por medio de una simulación Monte Carlo en Excel Visual Basic. En el método de Monte Carlo, para el cálculo de las opciones europeas en un mercado neutral al riesgo, el call y el put europeos están determinados como sigue:

$$
\begin{gathered}
C=E\left[\delta e^{-r T}\left(S e^{\sigma Z+\left(r-\frac{1}{2} \sigma^{2}\right) T}-K\right)^{+}\right] \\
P=E\left[\delta \alpha e^{-r T}\left(K-S e^{\sigma Z+\left(r-\frac{1}{2} \sigma^{2}\right) T}\right)^{+}\right]
\end{gathered}
$$

donde, $Z \sim N(0, T)$.

De la ecuación (11), se obtiene el valor de la opción mediante la aproximación de Monte Carlo.

$$
C=e^{-r T} \frac{1}{n} \sum_{i=1}^{n} C^{(i)}
$$

donde,

$$
C^{(i)}=\delta\left(S^{(i)}-K\right)^{+}
$$

De igual manera, se obtiene el valor del put europeo de la ecuación (12):

$$
P=e^{-r T} \frac{1}{n} \sum_{i=1}^{n} P^{(i)}
$$

Donde,

$$
C^{(i)}=\delta\left(S^{(i)}-K\right)^{+}(16)
$$

Se pueden expresar las precipitaciones acumuladas anuales $\mathrm{S}^{(\mathrm{i})}$ así:

$$
S^{(i)}=S_{0} e^{\sigma Z_{i}+\left(r-\frac{1}{2} \sigma^{2}\right) T}
$$

donde,

$$
Z_{i}=X_{i} \sqrt{T}(18)
$$

Cabe mencionar que $X_{i}$ corresponde a las variables aleatorias independientes generadas y distribuidas normalmente.

En la tabla 2, se presentan los parámetros usados para el cálculo de las opciones de cada estado. 
Aplicando la técnica de Monte Carlo ${ }^{18}$ con Excel Visual Basic, se realizaron diez mil iteraciones de las posibles trayectorias a un año, y se obtuvieron los resultados que se muestran en la tabla 3.

Tabla 2

Parámetros usados para el cálculo mediante el método Black-Scholes

\begin{tabular}{|c|c|c|c|}
\hline & Oaxaca & Chiapas & Veracruz \\
\hline $\mathrm{S}(\mathrm{mm})$ & 993.2 & $1,614.20$ & $1,614.20$ \\
\hline $\mathrm{K}(\mathrm{mm})$ & $1,343.83$ & $2,119.73$ & $1,618.84$ \\
\hline $\begin{array}{l}\text { Tasa de interés libre de riesgo } r \\
(31 / 12 / 2016)\end{array}$ & 0.0596 & 0.0596 & 0.0596 \\
\hline $\operatorname{Sigma}^{[1]}$ & 0.153 & 0.153 & 0.083 \\
\hline Vencimiento (año) & 1 & 1 & 1 \\
\hline $\begin{array}{l}\text { No. de trayectorias (iteraciones Monte } \\
\text { Carlo) }\end{array}$ & 10,000 & 10,000 & 10,000 \\
\hline (6) & $\begin{array}{l}10 \text { pesos } \\
/ \mathrm{mm}\end{array}$ & $\begin{array}{l}10 \text { pesos } \\
/ \mathrm{mm}\end{array}$ & $\begin{array}{l}10 \text { pesos } \\
/ \mathrm{mm}\end{array}$ \\
\hline
\end{tabular}

Fuente: Elaboración propia con datos de la Secretaría de Agricultura, Ganadería, Desarrollo Rural, Pesca y Alimentación de México y del Servicio de Información Agroalimentaria y Pesquera de México.

Tabla 3

Valor de la opción sobre precipitación

\begin{tabular}{lccc}
\hline & Chiapas & Veracruz & Oaxaca \\
\hline $\begin{array}{l}\text { Opción de compra europea sobre precipitación } \\
\text { (Call) }\end{array}$ & $\$ 0.00$ & $\$ 15.90$ & $\$ 0.00$ \\
$\begin{array}{l}\text { Opción de venta europea sobre precipitación } \\
(\text { Put })\end{array}$ & $\$ 494.70$ & $\$ 14.60$ & $\$ 343.92$ \\
Valor de la Prima del Call (pesos) & $\$ 0.00$ & $\$ 158.95$ & $\$ 0.00$ \\
Valor de la Prima del Put(pesos) & $\$ 4,946.96$ & $\$ 146.04$ & $\$ 3,439.22$ \\
\hline
\end{tabular}

Fuente: Elaboración propia con datos de la Secretaría de Agricultura, Ganadería, Desarrollo Rural, Pesca y Alimentación de México y del Servicio de Información Agroalimentaria y Pesquera de México, mediante Monte Carlo (i=10,000).

En la tabla 3, se observa que en los Estados de Veracruz y Chiapas no se pueden ofrecer opciones de compra, en virtud de que su prima es de cero, porque el valor de la precipitación de referencia es superior a la precipitación acumulada en el último año (2016). Sin embargo, si se supone que cada $\$ 1$ (unidad monetaria) corresponde a $1 \mathrm{~mm}$ de lluvia, entonces, se puede ofrecer

${ }^{18}$ Son prácticamente simulaciones dinámicas de las precipitaciones, dada su volatilidad histórica. Se generan posibles trayectorias del comportamiento del activo en un periodo definido. 
una opción de venta al caficultor, donde las primas para los Estados de Oaxaca, Chiapas y Veracruz son de 344 pesos (MXN), 494.7 pesos (MXN) y 14.6 pesos $(\mathrm{MXN})$, respectivamente.

Por lo tanto, se necesita un strike de precipitación de 2,119.73 mm, 1,618.84 mm y 1,343.83 mm, para asegurar la producción del año 2017, en los estados de Chiapas, Veracruz y Oaxaca, respectivamente. Si las precipitaciones anuales acumuladas están por debajo de dicho valor, entonces el comprador del put ejercerá su opción. Se puede, así, cubrir a los caficultores de las variaciones de la precipitación que afecta su producción, ya que este instrumento no influye en la demanda de café, ni en sus precios.

\section{Conclusión}

Este trabajo propone una opción climática de venta (put) sobre las precipitaciones, para los estados de Veracruz, Chiapas y Oaxaca. Por lo tanto, se presentaron los derivados climáticos y su aplicación en la agricultura, donde se encontró que la variabilidad del clima y el cambio climático afectan de manera significativa tanto la calidad, como la cantidad de la producción. Después, se presentó de manera breve, la situación de la producción de café en México y se encontró que prácticamente $80 \%$ de la producción de café proviene de los estados de Veracruz, Chiapas y Oaxaca. Para identificar cuál evento climático afecta en mayor medida la producción del café (si las precipitaciones o la temperatura), se utilizó un análisis econométrico para determinar el rendimiento de la producción de café.

Así, mediante una función de producción Cobb-Douglas, adaptada a dichos eventos, se realizó el estudio con un panel de datos, en el cual se encontró que hay una relación elástica entre precipitación $(P)$ y producción $(Y)$, lo que no sucede para el caso de la temperatura $(T)$. Se observó, también, que hay diferencia sistemática entre los efectos fijos y los efectos aleatorios, por lo que el modelo de efectos fijos es adecuado porque da el mejor ajuste de panel. Por lo tanto, las variaciones en la producción del café en los estados estudiados suceden en función de las precipitaciones, pero no de la temperatura. La temperatura no es un elemento determinante en la producción del café, contrariamente a la literatura sobre el café. Esta situación puede ser el resultado de la metodología utilizada o de la cantidad de datos utilizados.

Después de determinar, entre la temperatura y la precipitación, que afecta los rendimientos del café, se utilizó el método de Black-Scholes con una simulación Monte Carlo (10,000 iteraciones) para calcular tres opciones put sobre la precipitación, a fin de transferir el riesgo de los caficultores de Veracruz, Chiapas y Oaxaca. Con los datos del Banco de México, de SIAP y 
de CONAGUA, se obtuvo, después de la valuación, una prima de la opción de venta de 394.7 pesos (MXN) para el estado de Veracruz, 344 pesos (MXN) para el estado de Chiapas y 14.6 pesos (MXN) para el estado de Oaxaca.

Por lo tanto, los caficultores pueden contar con un derivado sobre la precipitación que proporciona una cobertura de su producción. Dichos resultados hacen posible enfrentar los diversos riesgos climáticos que afectan a diferentes agentes económicos, con unas coberturas económicas eficientes. Cabe señalar que estas opciones, como todos los derivados del clima, pueden ejercerse aunque no haya una causa directa en la variabilidad de la producción del café. Usarlos es asegurarse de la causa, y no de los efectos, provocados en la variabilidad del clima en la producción de un bien, mientras que el uso de los derivados y los seguros convencionales permite asegurarse, o cubrirse, de los efectos.

Se recomienda fomentar estudios financieros acompañados por meteorólogos, para profundizar en los modelos de predicción, con el fin de mejorar la medición del riesgo y reducir las potenciales pérdidas, y, así, dar el primer paso para su utilización en la agricultura mexicana.

\section{Referencias}

[1] Agrawala, Shardul (2005). "Bridge Over Troubled Waters. Linking Climate Change and Development". Organization for Economic Cooperation and Development. OECD:Francia.

[2] Alaton, P., Djehiche, B., \& Stillberger, D. (2002). "On modelling and pricing weather derivatives". Applied Mathematical Finance, 9(1), 1-20.

[3] Améndola, R., Castillo, E., \& Martínez, P. A. (2005). Country Pasture/Forage Resource Profiles. [CDROM]. México: FAO Roma.

[4] BANXICO (2018). "Estadística de CETES 91". Banco de México. México: Banco de México. Disponible en: www.banxico.org.mx

[5] Barnett, B. y Mahul, C. (2007). "Weather index insurance for agriculture and rural areas in lower income countries". American Journal of Agriculture Economics, 89 (5), 1241-1247.

[6] Benth, F.E. y Saltyte Benth, J. (2007). "The volatility of temperature and pricing of weather derivatives". Quantitative Finance, 7(5), 553-561.

[7] Black, F., y Scholes, M. (1973). "The Pricing of Options and Corporate Liabilities". Journal of Political Economy, 81(3), 637-654.

[8] Cruz, J. S., y Llinás, A. (2010). "Modelo Analítico de Derivados de Clima para Eventos Específicos de Riesgo en la Agricultura en Colombia”. Cuadernos de Desarrollo Rural, 7 (64), 121-147.

[9] Cruz, J., y Vargas, C. (2008). "Pricing de un exótico del clima para Colombia". Disponible en: www. cesa. edu. co

[10] Dang, G. (2006). Agriculture, Rural Areas and Farmers in China. China Intercontinental Press: Beijing. 
[11] Easterling, D.R., Evans, J.L., Groisman, P.Y., Karl, T.R., Kunkel, K.E. y Ambenje, P. (2000). "Observed variability and trends in extreme climate events: a brief review". Bulletin of the American Meteorological Society, 81(3), 417-426.

[12] Fuchs, A. y Wolff, H. (2011). "Concept and unintended consequences of weather index insurance: the case of Mexico". American Journal of Agricultural Economics, 93(2), 505-511.

[13] Gay, C., Estrada, F., Conde, C., Eakin, H., y Villers, L. (2006). "Potential Impacts of Climate Change on Agriculture: A Case of Study of Coffee Production in Veracruz. México, DF, México". Climatic Change, 79(3-4), 259-288. DOI:10.1007/s10584-006-9066-x

[14] González Sánchez, M., Nave Pineda, J.M. (2010). "Valoración de derivados sobre el clima a partir de la modelización estocástica de la temperatura en el Aeropuerto El Dorado de Bogotá”. Cuadernos de Administración, 23 (41), 261-283.

[15] Gronberg, Timothy J., y Neilson, William S. (2007). Incentive under Weather Derivatives vs. Crop Insurance. (Unpublished Manuscript). Institute for Science, Technology and Public Policy, Texas A\&M University.

[16] GTZ (2010). "Sociedad para la Cooperación Técnica, dependiente del Ministerio Federal de Cooperación Económica y Desarrollo y Café Direct. Cambio Climático y Café: Capacitación para productores y organizaciones cafetaleras". Gesellschaft für Technische Zusammenarbeit. Eschborn, Alemania.

[17] Houghton, J.T., Ding, Y., Griggs, D.J., Noguer, M., van der Linden, P.J., Dai, X., Maskell, X., y C.A. Johnson (2001). "Contribution of working group I to the third assessment report of the intergovernmental panel on climate change". Climate Change, The Scientific Basis, 388. Cambridge University Press.

[18] Jaramillo, J., Muchugu, E., Vega, F., Davis, A., Borgemeister, C. y Chabi-Olaye, A. (2011). "Some Like It Hot: The Influence and Implications of Climate Change on Coffee Berry Borer (Hypothenemushampei) and Coffee Production in East Africa". Plos One (9): e24528. DOI:10.1371/journal.pone.0024528.

[19] Jewson, S. y Brix, A. (2005). Weather Derivative Valuation: The Meteorological, Statistical, Financial and Mathematical Foundations. Cambrigde University Press: New York.

[20] Moguel, P., y Toledo, V.M. (1999)."Biodiversity conservation in traditional coffee systems of Mexico." Conservation Biology 13.1,11-21.

[21] Parry, M.L., Canziani, O.F., Palutikof, J.P., van der Linden, P.J. y Hanson, C.E. (eds.) (2007). Climate change 2007: impacts, adaptation and vulnerability. Contribution of working group II to the fourth assessment report of the Intergovernmental Panel on Climate Change. Vol. 4. Cambridge University Press: E.U.A.

[22] Paz Cobo, S. (2004). “Derivados vinculados al seguro". Universidad Pontificia de Salamanca. Madrid, España.

[23] Rosenzweig, C., Iglesias, A., Yang, X.B., Epstein, P.R. y Chivian, E. (2001), "Climate change and extreme weather events; implications for food production, plant diseases, and pests". Global Change and Human Health 2(2), 90-104. 
[24] Schiller, F., Seidler, G. y Wimmer, M. (2012). "Temperature models for pricing weather derivatives". Quantitative Finance, 12(3), 489-500.

[25] SAGARPA (2015). "Reporte informativo sobre los impactos del café". Secretaría de Agricultura, Ganadería, Desarrollo Rural, Pesca y Alimentación, México: SAGARPA. Disponible en: www.gob.mx/sagarpa

[26] SAGARPA (2017). "Cierre de la Producción Agrícola". Secretaría de Agricultura, Ganadería, Desarrollo Rural, Pesca y Alimentación, México: SAGARPA. Recuperado de http://infosiap.siap.gob.mx

[27] SMN (2018). "Resúmenes mensuales de temperaturas y lluvia". Servicio Meteorológico Nacional, México: SMN. Recuperado de: http://smn.cna.gob.mx

[28] SIAP (2014). "Sistemas Producto Café". Servicio de Información Agroalimentaria y Pesquera. México: SIAP. Recuperado de http://w4.siap.gob.mx/sispro

[29] Stoppa, A. y Hess, U. (2003). "Design and use of weather derivatives in agricultural policies: the case of rainfall index insurance in Morocco." International Conference: Agricultural Policy Reform and the WTO: Where are We Heading? Capri (Italy).

[30] Turvey, C.G. (2001). "Weather Derivatives for Specific Event Risks in Agriculture." Review of Agricultural Economics, 23(2), 333 - 351.

[31] Vaast P, Bertrand B, Perriot J-J, Guyot B, Genard M (2006). "Fruit thinning and shade improve bean characteristics and beverage quality of coffee (Coffea arabica L.) under optimal conditions." Journal of the Science of Food and Agriculture 86(2), 197-204.

[32] Vedenov, D.V. y Barnett, B.J. (2004). "Efficiency of weather derivatives as primary crop insurance instruments". Journal of Agricultural and Resource Economics, 29(3), 387-403.

[33] Villers, L., N. Arizpe, R. Orellana, C. Conde y J. Hernández (2009). "Impactos del cambio climático en la floración y desarrollo del fruto del café en Veracruz, México". Interciencia 34(5), 322-329.

[34] Wang, H.H., Karuaihe, R.N., Young, D.L. y Zhang, Y. (2013). "Farmers' demand for weather based crop insurance contracts: the case of maize in South Africa." Agrekon, 52(1), 87-110. 